\title{
Arenas calizas para la confección de hormigones: influencia de sus finos calizos y arcillosos a nivel de morteros
}

\author{
J. L. RAMIREZ ORTIZ, Prof. Dr. Ing. Industrial \\ J. M. BARCENA DIAZ, Dr. Ing. Industrial \\ J. I. URRETA ORMAECHEA, Ing. Industrial
}

LABORATORIO DE ENSAYOS E INVESTIGACIONES INDUSTRIALES

"L. J. Torróntegui”. LABEIN (Bilbao)

\section{$R E S U M E N$}

Con el propósito de obtener datos para una próxima revisión de la Norma Española de Hormigón en cuanto al contenido en finos en arenas, actualmente fijado sin excepción en el $5 \%$, se ha abordado un proyecto de investigación bastante exhaustivo. Se presentan a continuación los resultados de la influencia de diferentes contenidos de finos calizos y arcillosos en las propiedades mecánicas de los morteros, paso previo al estudio de dicha influencia en hormigones confeccionados con árido calizo de machaqueo, fase actualmente en ejecución. Previamente a estos trabajos experimentales se realizó una prospección general de todas las canteras de machaqueo de caliza en el área norte de la península, correspondiente al País Vasco. Por los resultados obtenidos parece que el límite podrá elevarse hasta el 12-15\%, siempre que se garantice una presencia de arcilla limitada por ensayos.

\section{$S U M M A R Y$}

For the purpose to obtain data for a next revision of the Spanish Concrete Norm about the content of fines in sands fixed to date on $5 \%$ without exceptions, an exhaustive research project has been undertaken.

Following, the results of the influence of different contents of calcareous and clayey fines in mortar mechanical properties, are shown, first step to begin the study of this influence on concretes made with limestone aggregate, presently in execution phase. Before those experimental works, a general survey at all the limestone aggregate quarries in northern area of the peninsula corresponding to the Basque Country, was accomplished. By the results obtained it seems that it will be possible to enlarge the limit untill 12-15\%, if only a limited presence of clay is assured.

\section{PROPOSITO}

Un objetivo de este trabajo es estudiar la influencia que contenidos variables de finos calizos y arcillosos, procedentes del machaqueo de piedra caliza, introducen en algunas de las características del hormigón.

Otro de los objetivos es suministrar datos y propuestas relativas a la modificación del contenido admisible de finos en las arenas que desea introducirse en la Instrucción Española para obras de hormigón EH-82.

\section{FASES DE TRABAJO}

El programa de investigación se ha dividido en las siguientes fases:

$\left.1 .^{a}\right)$ Estudio de las canteras de machaqueo del Pais Vasco. 
2. ${ }^{a}$ ) Influencia del contenido de finos en morteros de cemento.

$3{ }^{a}$ ) Repercusión del contenido de finos en hormigones.

La primera fase está concluida y publicada, consistiendo en el estudio de 44 canteras, con determinación de sus características geológicas, físicas y químicas y las de los áridos que fabrican para la confección de morteros y hormigones (1) (2).

Esto ha servido para conocer el material de que se dispone de cara a dar al estudio un planteamiento realista. Como resultado de esta fase del trabajo se ha comprobado que el límite del $5 \%$ de finos en las arenas, de la instrucción EH-82 es superado ampliamente en todas las canteras visitadas, con la excepción de dos que disponen de hidrociclón y pueden preparar arenas dentro de normas.

El trabajo que se presenta en esta ocasión es la 2." fase: "Influencia del contenido de finos calizos y arcillosos en la resistencia de morteros de cemento".

Están muy avanzados los trabajos de la $3 .^{a}$ fase relativa a la repercusión en hormigones, que se espera tener concluido a finales de 1985. Se han manejado hasta el momento 14 toneladas de árido, con la confección de cientos de probetas normalizadas de hormigón.

La financiación de este trabajo corre a cargo del Gobierno de la Comunidad Autónoma del Pais Vasco.

\section{DESARROLLO DE LOS TRABAJOS SOBRE MORTEROS}

El objetivo de esta fase es doble: por un lado estudiar la influencia de los finos calizos y arcillosos en las características de los morteros y, por otro, establecer una base más estrecha para centrar con mejor conocimiento su influencia en los hormigones (3).

De las numerosas variables que intervienen en el problema se han puesto en juego tres, que han sido: granulometría de la arena, contenido en finos calizos y contenido en finos arcillosos. Se han mantenido fijas la dosificación y plasticidad de las masas.

Para evitar variabilidad en las arenas se ha procedido a separar elementos puros: árido, finos calizos y finos arcillosos, y mezclarlos de diversas maneras con objeto de recomponer los diferentes tipos de arenas a ensayar.

Las características de los morteros que se han evaluado han sido las resistencias a flexotracción y compresión a los 28 días de edad.

\subsection{Granulometría de la arena}

Para establecer la granulometria de la arena más conveniente se ha partido de los datos obtenidos en el estudio realizado de todas las canteras existentes en el País Vasco (2).

Ante la variedad de curvas granulométricas observadas en la arena suministrada por las canteras, se han elegido dos mediante los criterios siguientes:

Para la granulometría menos gruesa se ha tomado la más fina de las encontradas en la prospección de canteras, para tener datos sobre el valor extremo y, sobre todo, por estar dentro de los husos granulométricos propuestos por la norma americana A.S.T.M. C 33-82 y la francesa NF.P 18-301. 
Para la granulometría gruesa no se ha tomado el extremo más grueso encontrado en nuestra prospección, por salirse de los husos citados, Se ha elegido la más gruesa compatible con la norma más tolerante a este respecto que es la A.S.T.M.

De esta manera se ensayan los dos extremos granulométricos encontrados en el País Vasco, compatibles con normas internacionales.

Una vez elegidas estas granulometrias, se les ha eliminado la fracción más fina (menor de $80 \mu$ ) para poder recomponer las arenas con aportaciones variables de finos.

Los husos granulométricos resultantes de estas dos arenas así definidas, teniendo en cuenta el abanico del contenido en finos empleado en cada caso, puede verse en el gráfico 1.

\subsection{Contenido en finos totales de las arenas}

Analizando el contenido en finos de las canteras estudiadas (2), se han obtenido valores comprendidos entre el 7 y el $23 \%$ siendo los valores más frecuentes los comprendidos entre el 10 y el $12 \%$.

Tomando en cuenta estos valores y el limite normativo del $5 \%$ se optó por los siguientes contenidos de finos a utilizar en la recomposición de arenas:

\begin{tabular}{lllllll} 
& \multicolumn{6}{c}{ Finos \% } \\
\cline { 2 - 6 } & 3, & 5, & 8, & 12, & 15 \\
Granulometría gruesa: & 3, & 5, & 8, & 12, & 15, & 20
\end{tabular}

(1) Huso granulométrico de la arena fina.

(2) Huso granulometrico de la arena gruesa.

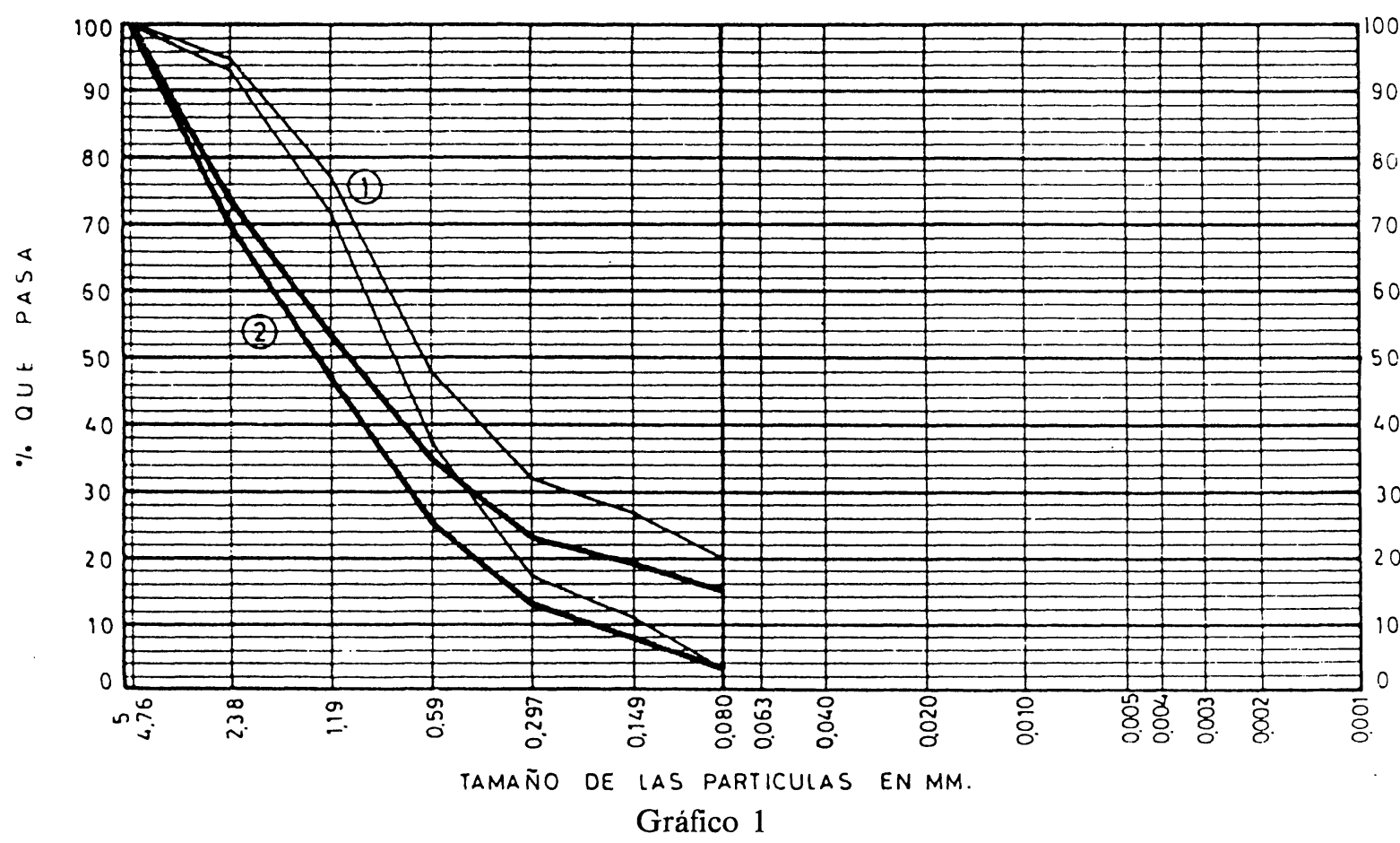


La razón en añadir el $20 \%$ para la granulometria fina es debido a que por lo general las granulometrías más finas también tienen mayor contenido de finos, debido a su proceso de elaboración.

\subsection{Contenido en finos arcillosos de las arenas}

La arcilla es el contaminante de las arenas calizas de machaqueo. Esta arcilla proviene de los restos de las monteras de las canteras que caen durante las voladuras y de los arrastres que produce la lluvia. Según los análisis realizados, resulta que el contenido de arcilla en las canteras de caliza investigadas no sobrepasa, en general, el valor del $2 \%$ respecto de la arena. Para recomponer la arena con proporciones variables de arcilla se intentó obtener arcilla menor de $2 \mu$, procedente de las monteras. El problema es que el material de las monteras está muy mezclado con piedras y restos vegetales, por lo que para obtener por sedimentación partículas menores de $2 \mu$, hubiera sido necesario tratar una gran cantidad de material. Esta dificultad se agravaría llegando a ser prácticamente imposible de resolver en la fase de elaboración de hormigones, donde los volúmenes de áridos son mucho mayores. Por ello se decidió obtener arcilla de tamaño menor de $40 \mu$ de otra fuente que no fuera de las monteras de las canteras, pero que tuviera una composición mineralógica parecida y el mayor contenido posible de partículas menores de $2 \mu$. Esta otra fuente fue la arcilla de las balsas de decantación de una cantera silicea.

El estudio mineralógico se realizó por difractometría de rayos $\mathrm{X}$ obteniéndose los siguientes resultados:

\begin{tabular}{|c|c|c|c|c|}
\hline & Illita & Vermiculita & Montmorillonita & Caolinita \\
\hline Arcilla de montera $<2 \mu(\%)$ & 84 & 15 & 1 & - \\
\hline Arcilla de balsa $<40 \mu(\%)$ & 88 & - & - & 11 \\
\hline Arcilla de balsa $<2 \mu(\%)$ & 89 & - & - & 11 \\
\hline
\end{tabular}

Aunque las arcillas no son iguales, sí son parecidas y además los componentes de tipo montmorillonítico, que poseen una gran capacidad de adsorción de agua y por tanto influencia en la plasticidad de las masas están prácticamente ausentes en ambas.

En resumen hemos tomado en este trabajo como arcilla el material menor que $40 \mu$ proviniente de las balsas de decantación.

La granulometría de esta arcilla puede verse en el gráfico 2.

Para el contenido de arcilla en las arenas se ha utilizado una banda de valores que alcanza un máximo del $6 \%$, aunque este valor sea excesivo, con el fin de que puedan ponerse de manifiesto las tendencias con mayor claridad.

En el momento que el ensayo del "azul de metileno" esté puesto a punto, se podrá comparar la actividad de las arcillas de las canteras con la de la balsa $(<40 \mu)$ utilizada en el ensayo y asi se conocerá el grado de equivalencia de los porcentajes de arcilla empleados en los ensayos y observados en los áridos. Por el momento suponemos que son equivalentes.

\subsection{Tipos de arenas recompuestas}

Con los criterios anteriormente expuestos se decidió estudiar los tipos de arenas de los cuadros 1 y 2 . 
Curva granulométrica de la arcilla de Gorocika.

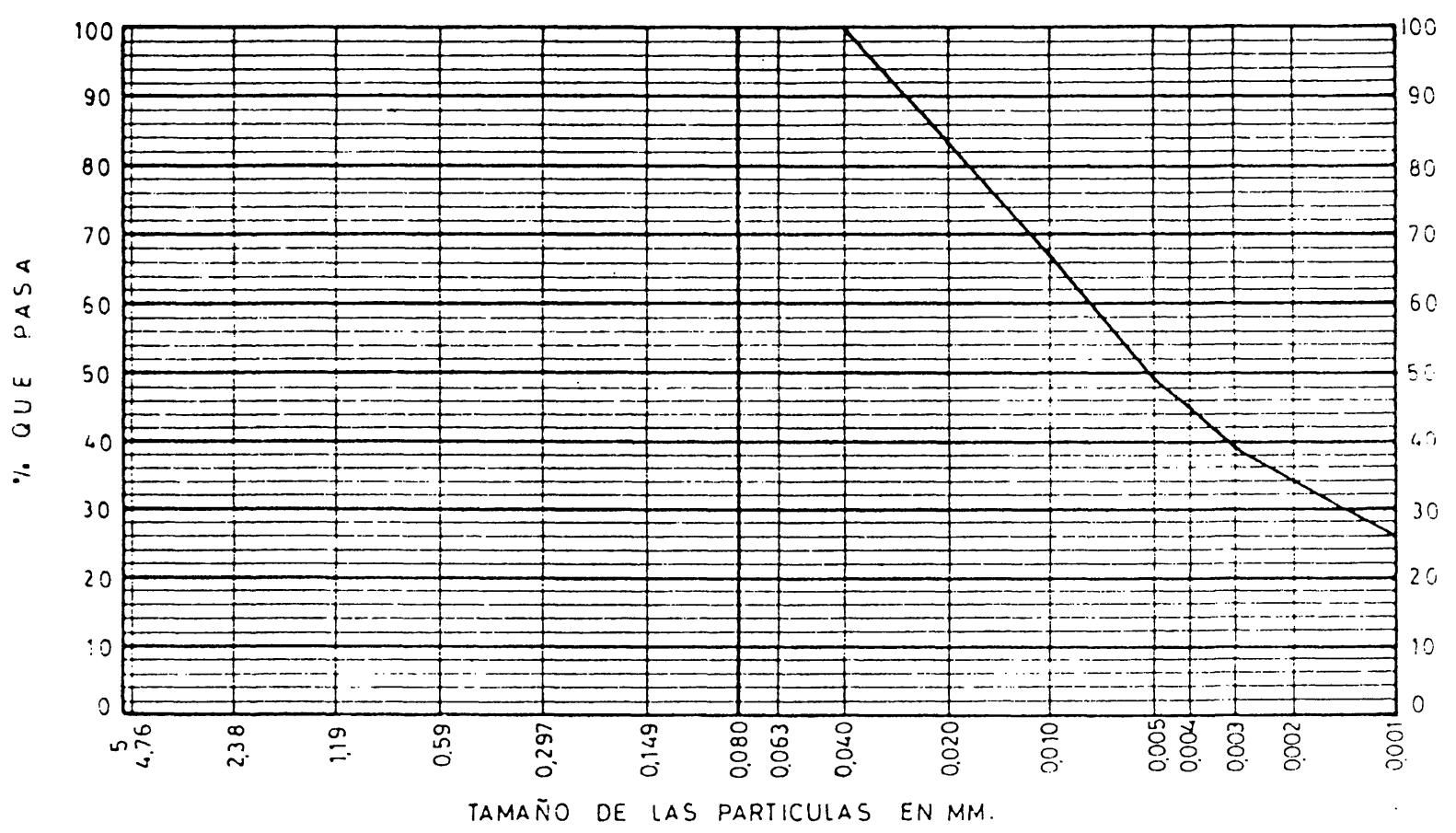

Gráfico 2

\section{UADRO 1 \\ ARENA FINA}

Contenido de arcilla en la arena (\%)

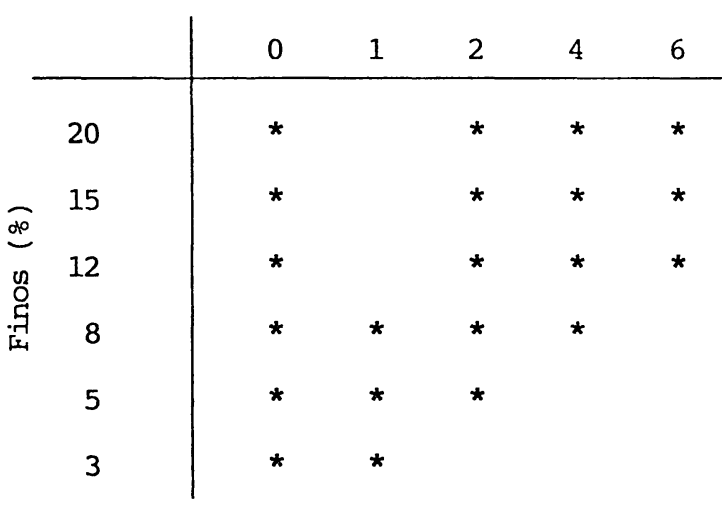

C UADRO 2

ARENA GRUESA

Contenido de arcilla en la arena (\%)

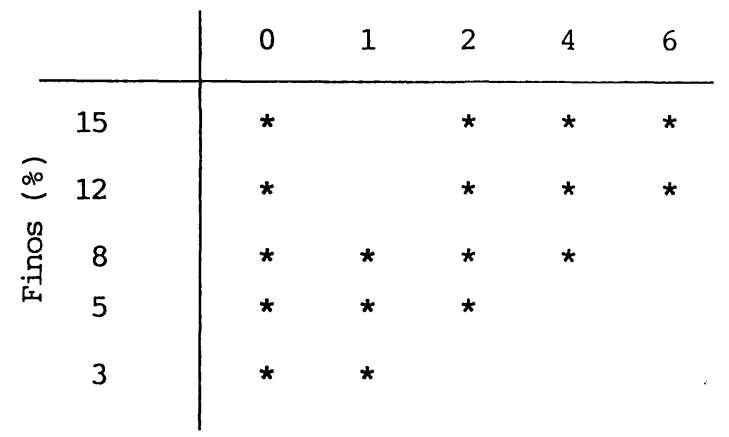

\section{RECOMPOSICION DE LAS ARENAS}

\subsection{Materiales}

Los materiales empleados fueron: árido calizo puro desprovisto de todo tipo de finos menores de $80 \mu$, finos calizos puros menores de $80 \mu$ y finos arcillosos menores de $40 \mu$.

\subsubsection{Arido calizo}

Para obtener este material lo más puro posible se eligió una de las canteras estudiadas, que tuviera una roca de alto contenido en $\mathrm{CO}_{3} \mathrm{Ca}$, sin montera arcillosa y con instalaciones adecuadas para el lavado y tratamiento de áridos. 
En esta cantera se tomaron varias toneladas de "balasto" (árido de $60 \mathrm{~mm}$.), que fueron lavados bajo ducha enérgica en la caja inclinada de un camión hasta que el agua de lavado resultara totalmente limpia.

Dicho material se trituró en una instalación limpiada previamente, desechándose el primer tercio producido para tener más garantías de la pureza obtenida.

De esta manera se obtuvieron $700 \mathrm{~kg}$. de arena teóricamente carente de arcilla contaminante, que fue tratada en laboratorio mediante tamizados y lavados, hasta obtener 6 fracciones de arena perfectamente desprovistas de toda clase de finos $(<80 \mu)$.

Las fracciones obtenidas fueron las comprendidas entre los tamices de luz de malla $(\mathrm{mm})$ siguientes:

$$
\begin{array}{ll}
4,76 & -2,38 \\
2,38 & -1,19 \\
1,19 & -0,59 \\
0,59 & -0,297 \\
0,297 & -0,149 \\
0,149 & -0,080
\end{array}
$$

Con esta operación se pudo disponer del material base calizo puro necesario para recomponer los tipos de arenas antes mencionados.

\subsubsection{Contaminante arcilloso}

Se tomo este material de las balsas de decantación, resultante del lavado de áridos silíceos. Los fangos se secaron primeramente en estufa y posteriormente se pulverizaron con escaso aporte de energia para no fracturar las partículas. Finalmente se tamizó el material resultante por el tamiz UNE 0,040 para obtener el contaminante arcilloso menor de $40 \mu$, necesario para recomponer las arenas.

\subsubsection{Finos calizos}

Estos finos se obtuvieron del proceso de tratamiento del árido calizo antes descrito.

Como el "balasto calizo" se lavó perfectamente antes de su machaqueo, los finos obtenidos en el machaqueo y molienda estaban exentos de arcilla. Estos finos se consiguieron por tamizado en seco del balasto machacado.

\subsection{Mezclado}

Con las diferentes fracciones preparadas se recompusieron las granulometrias gruesas y finas, con los contenidos de finos calizos y arcillosos señalados en los cuadros 1 y 2 .

El método operatorio fue el siguiente: se tomaron las fracciones de árido necesario junto con los finos calizos y arcillosos, y se introdujeron en un recipiente cilindrico de plástico que giraba lentamente alrededor de su eje. De esta forma se obtenia una mezcla intima de las 7 fracciones de material calizo utilizado y de los finos arcillosos.

Para conseguir que las partículas finas (calizas y arcillosas) se adhirieran a las más gruesas, tal como se puede observar en el árido natural, se procedía a añadir agua, equivalente en un $2,5 \%$ 
en peso de la muestra. Para ello se vertia la muestra en una bandeja y utilizando un pulverizador se esparcía uniformemente el agua necesaria. Al mismo tiempo se realizaba un mezclado con una paleta para obtener una mayor uniformidad.

Esta comprobación de adherencia se ha realizado por medio de microscopio electrónico de barrido. Se ha podido apreciar que el aspecto final de las arenas recompuestas, resultaba muy similar a la arena natural, tanto por su aspecto visual como a través del microanálisis de las particulas finas adheridas a las maś gruesas.

Una vez obtenida la mezcla se la dejaba reposar 24 horas para una perfecta difusión de la humedad. Después de seca quedaba dispuesta para la operación de amasado.

\section{AMASADO DE LOS MORTEROS}

Para realizar esta operación se ha partido de los métodos, equipos de amasado, compactado y moldeo del "Pliego de Prescripciones Técnicas Generales para la Recepción de Cementos RC-75".

Se confeccionaron 38 masas de mortero de la misma dosificación (cemento/arena $=1 / 4,2$ en peso, equivalente a $430 \mathrm{~kg}$ de cemento/ $\mathrm{m}^{3}$ mortero).

Se utilizó la misma amasadora que se emplea para los ensayos de cemento, pero modificando las paletas, para que la holgura entre paleta giratoria y cuba fuera algo mayor. El objetivo de esta modificación fue el impedir el fraccionamiento del árido en el amasado, como consecuencia del mayor tamaño de esta arena $(5 \mathrm{~mm})$ en comparación con la que se usa en los ensayos de cemento.

Se pretendía que todas las masas tuvieran la misma manejabilidad y para fijarla se recurrió a la mesa de sacudidas del antiguo Pliego de cementos. El número de golpes de este ensayo se obtuvo en pruebas previas de manera que se dispusiera de una sensibilidad suficiente. Como conclusión de estas pruebas resultó que con un escurrimiento de $45 \pm 2 \mathrm{~mm}$ en 15 caídas de la mesa, se obtenía una plasticidad similar a la de un mortero de tipo plástico.

En el caso de no conseguir esta plasticidad al primer intento, se añadiria agua o una fracción de arena y cemento, proporcionando un amasado complementario. Este segundo amasado introduce una demora en el tiempo de amasado, con las pérdidas de agua consiguientes por evaporación y absorción del árido. Estos efectos fueron estudiados experimentalmente y corregidos en los resultados finales obtenidos.

\section{COMPACTACION, ENMOLDADO Y CURADO}

Se realizaron varias pruebas para determinar el número de golpes más conveniente en la mesa compactadora de los cementos, llegando a considerar como satisfactorio el mismo que figura en el ensayo normalizado.

Los moldes empleados fueron los del ensayo de cemento $(4 \times 4 \times 16 \mathrm{~cm})$ en número de 3 por cada amasada.

Todo el proceso de amasado, compactación, curado y ensayo a 28 dias de las probetas se realizó siguiendo las indicaciones del Pliego de cemento antes mencionado.

En el gráfico 3, se presenta un esquema general de todo el método operatorio correspondiente al trabajo que se expone. 


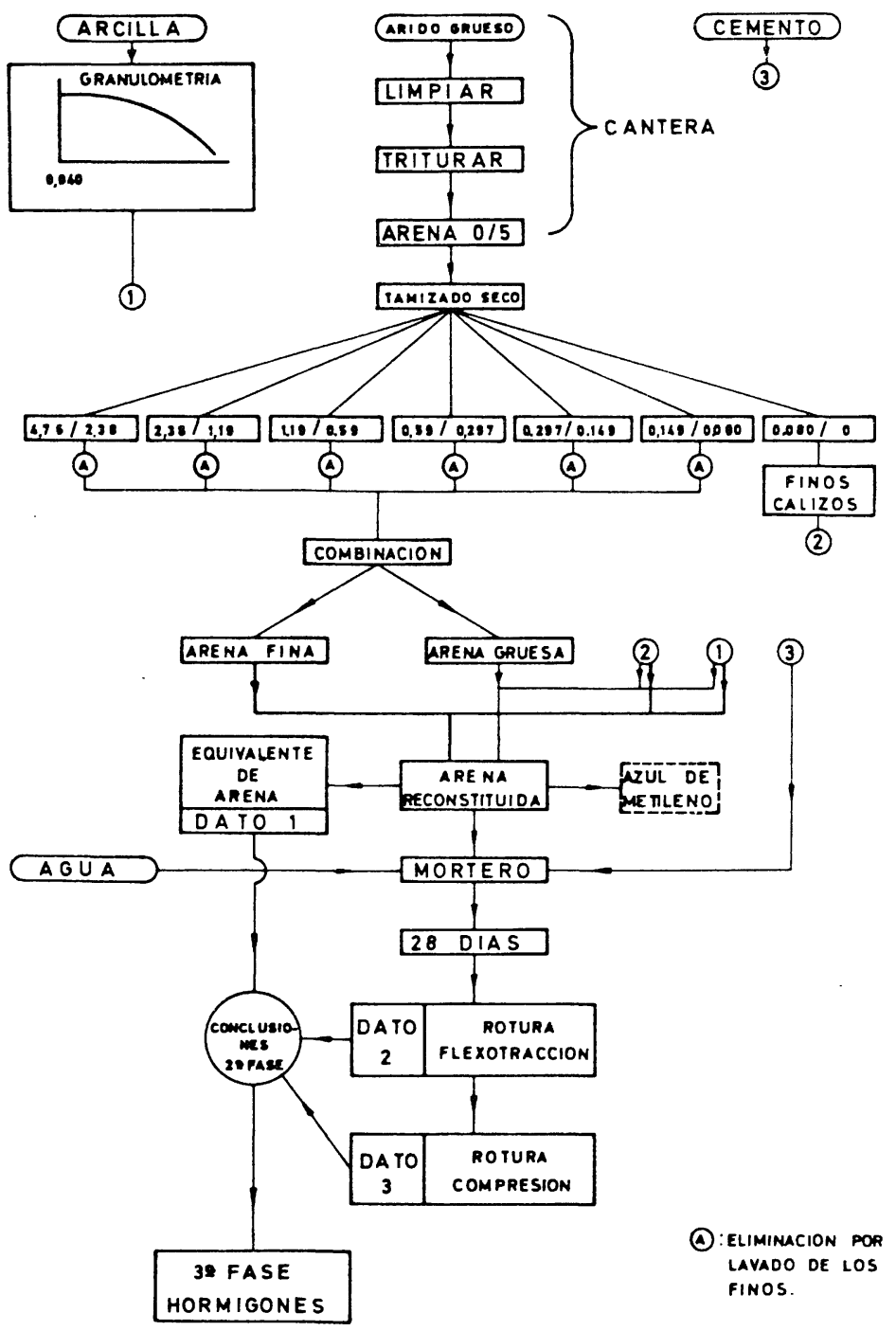

Gráfico 3

\section{RESULTADOS DE LOS ENSAYOS}

Los resultados obtenidos se han concretado en gráficos de dos dimensiones.

Para cada una de las dos granulometrias ensayadas se incluyen los siguientes gráficos:

Equivalente en arena - Contenido en finos (\%) (gráficos 4 y 5).

Relación A/C - Contenido en finos (\%) (gráficos 6 y 7).

Resistencia a compresión - Contenido en finos (\%) (gráficos 8 y 10).

Resistencia a compresión - Proporción Arcilla/Arena (\%) (gráficos 9 y 11).

Resistencia a flexotracción - Contenido en finos (\%) (gráficos 12 y 14).

Resistencia a flexotracción - Proporción Arcilla/Arena (\%) (gráficos 13 y 15). 


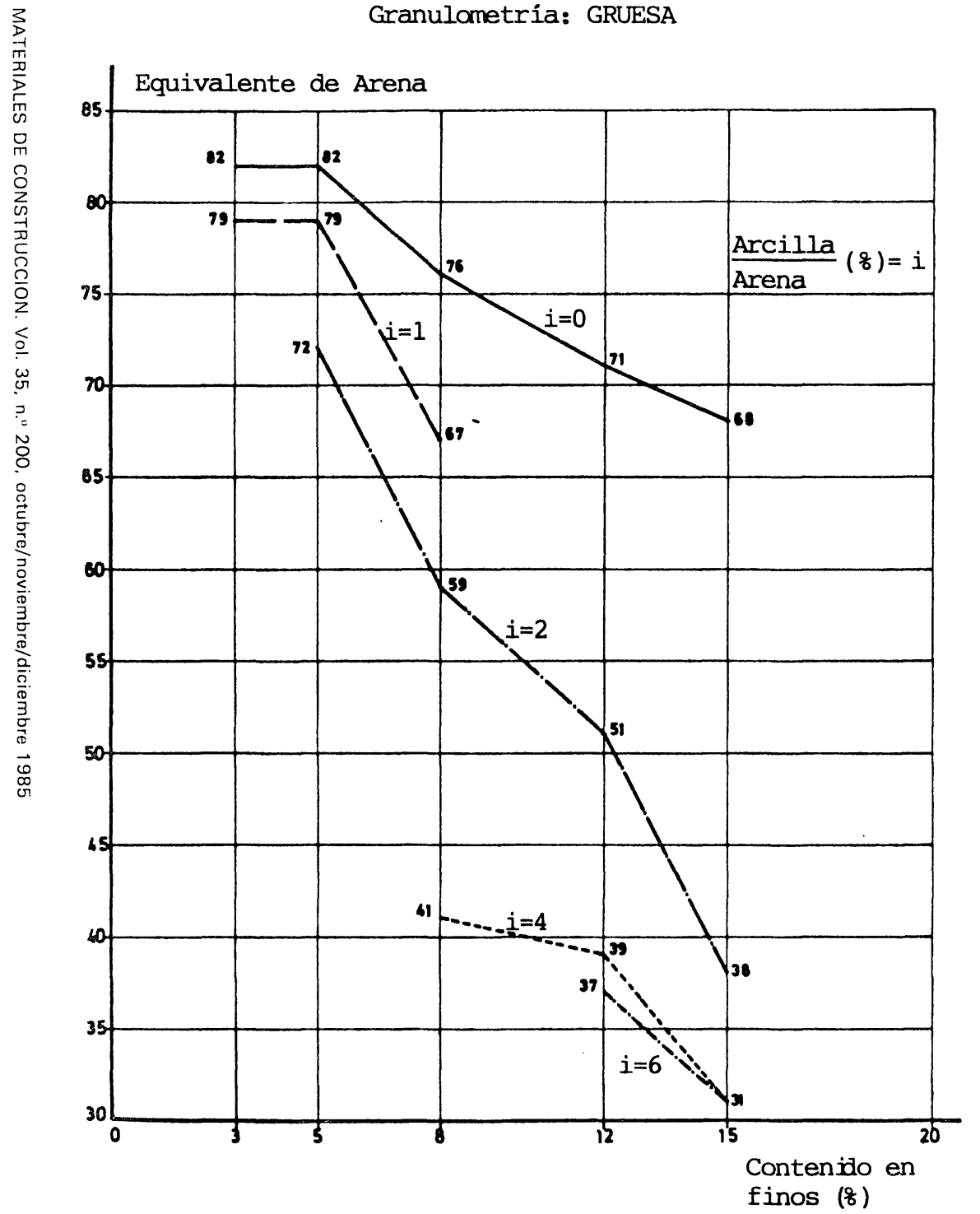

$\stackrel{\omega}{\omega}$
Granulometria: FINA

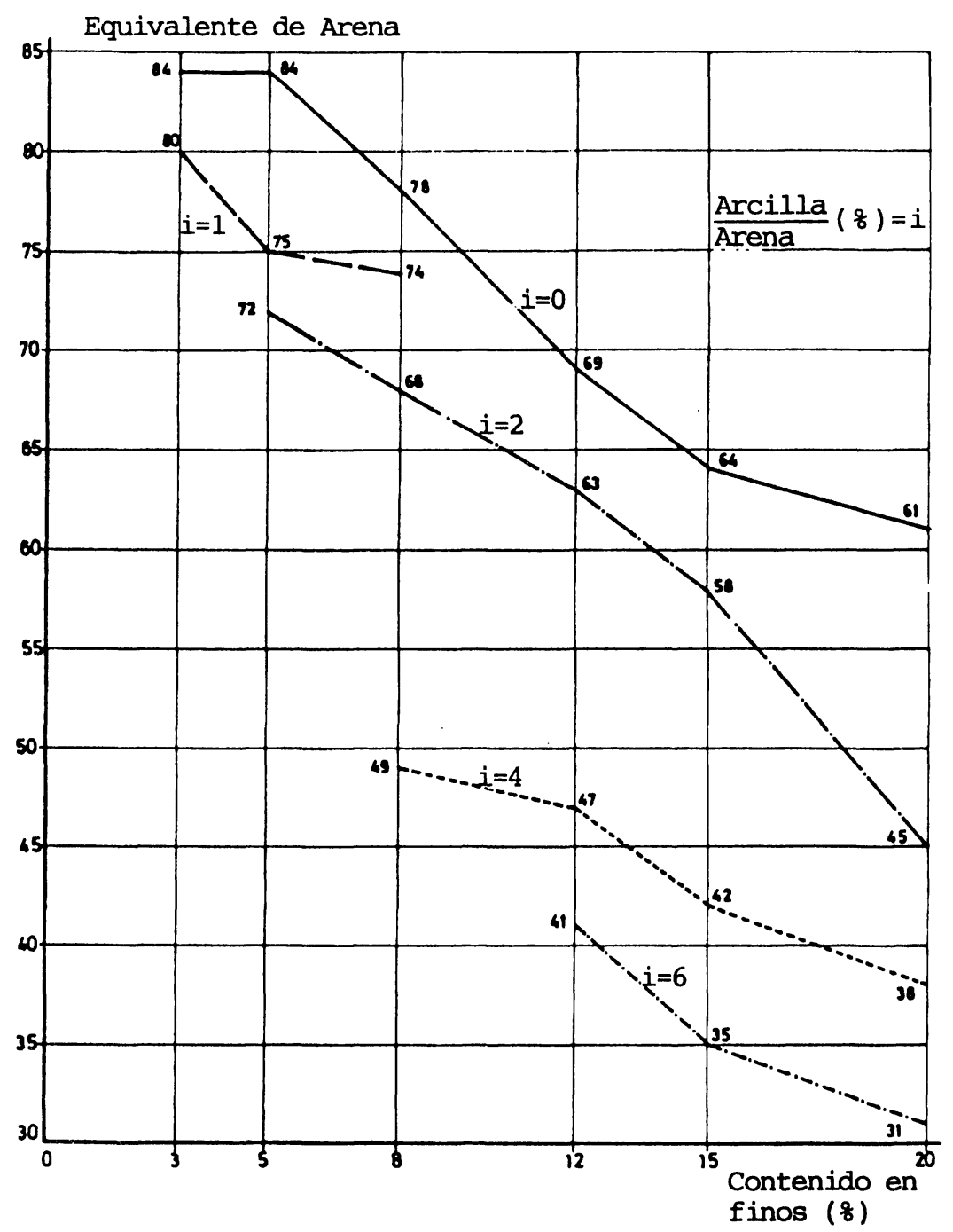

Gráfico 5 

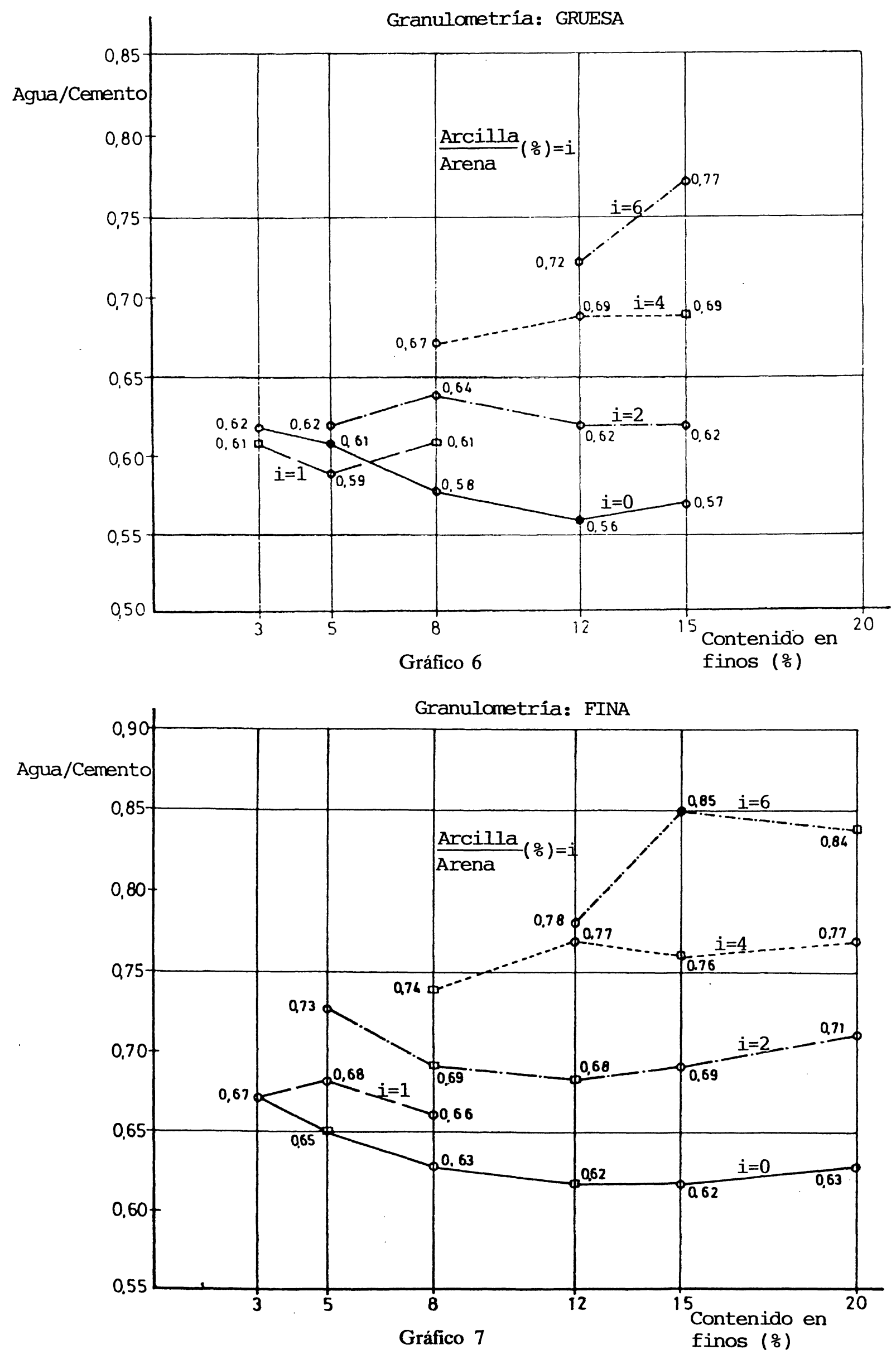

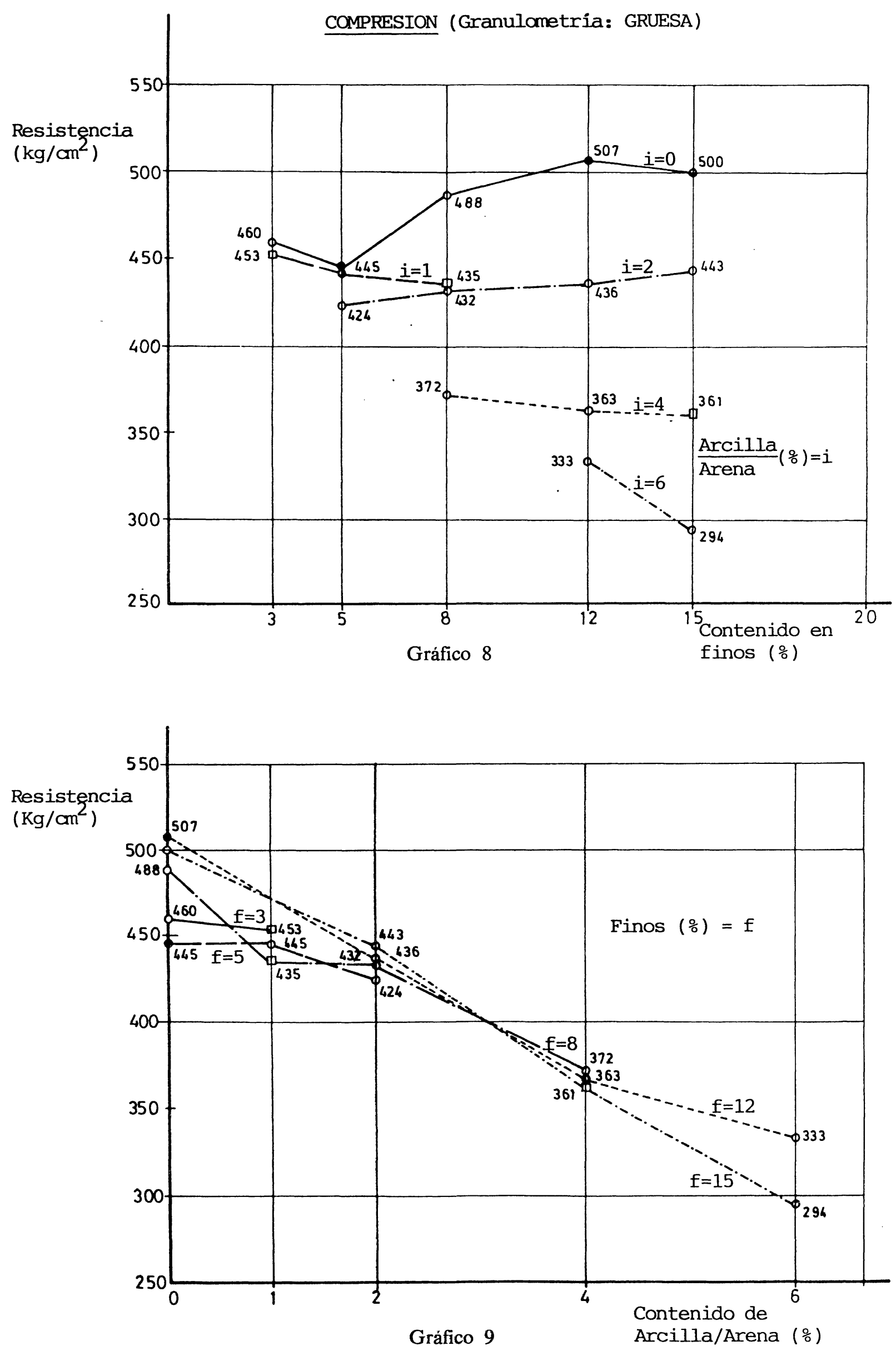

MATERIALES DE CONSTRUCCION. Vol. 35, n.²00, octubre/noviembre/diciembre 1985 

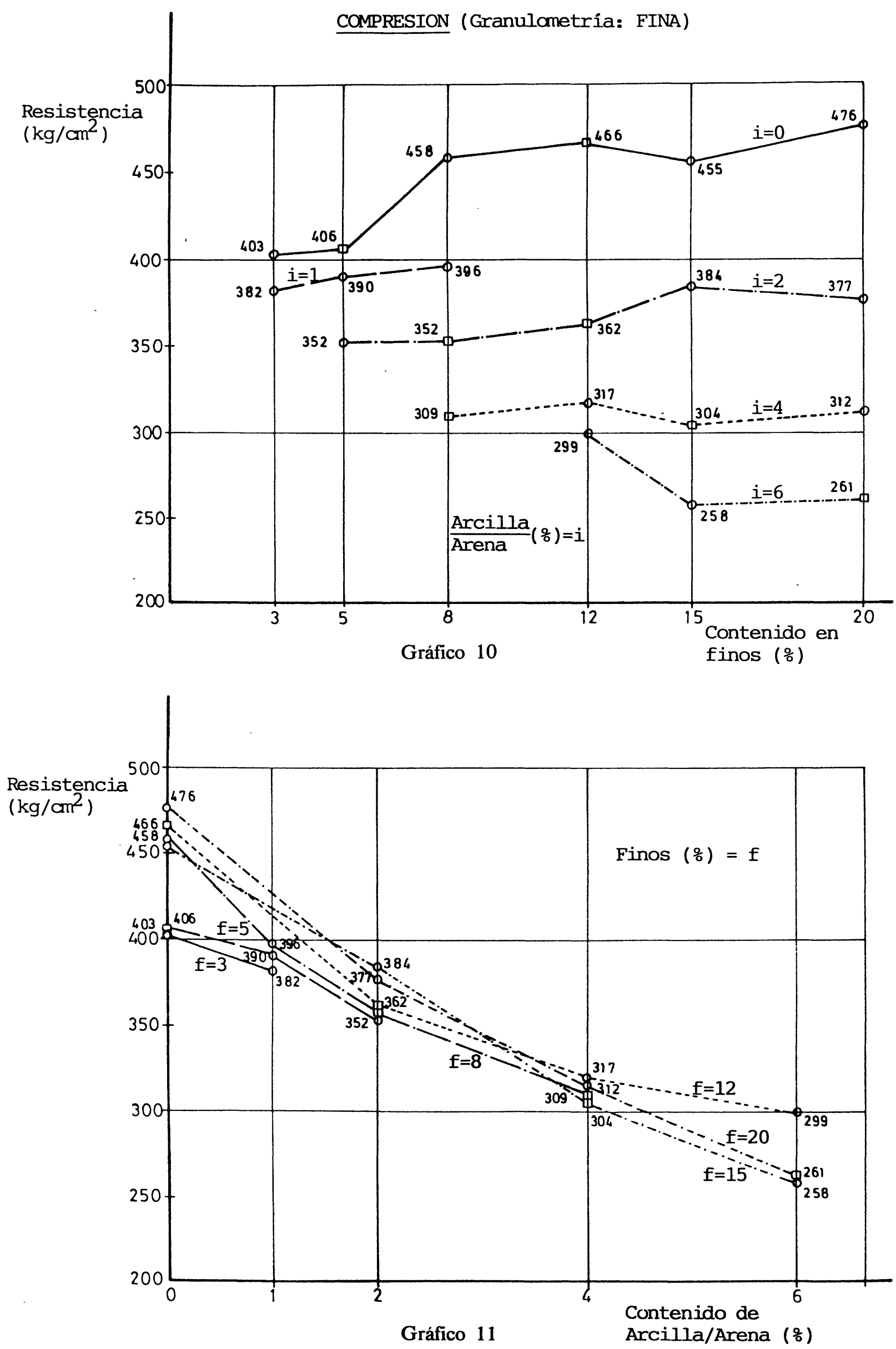

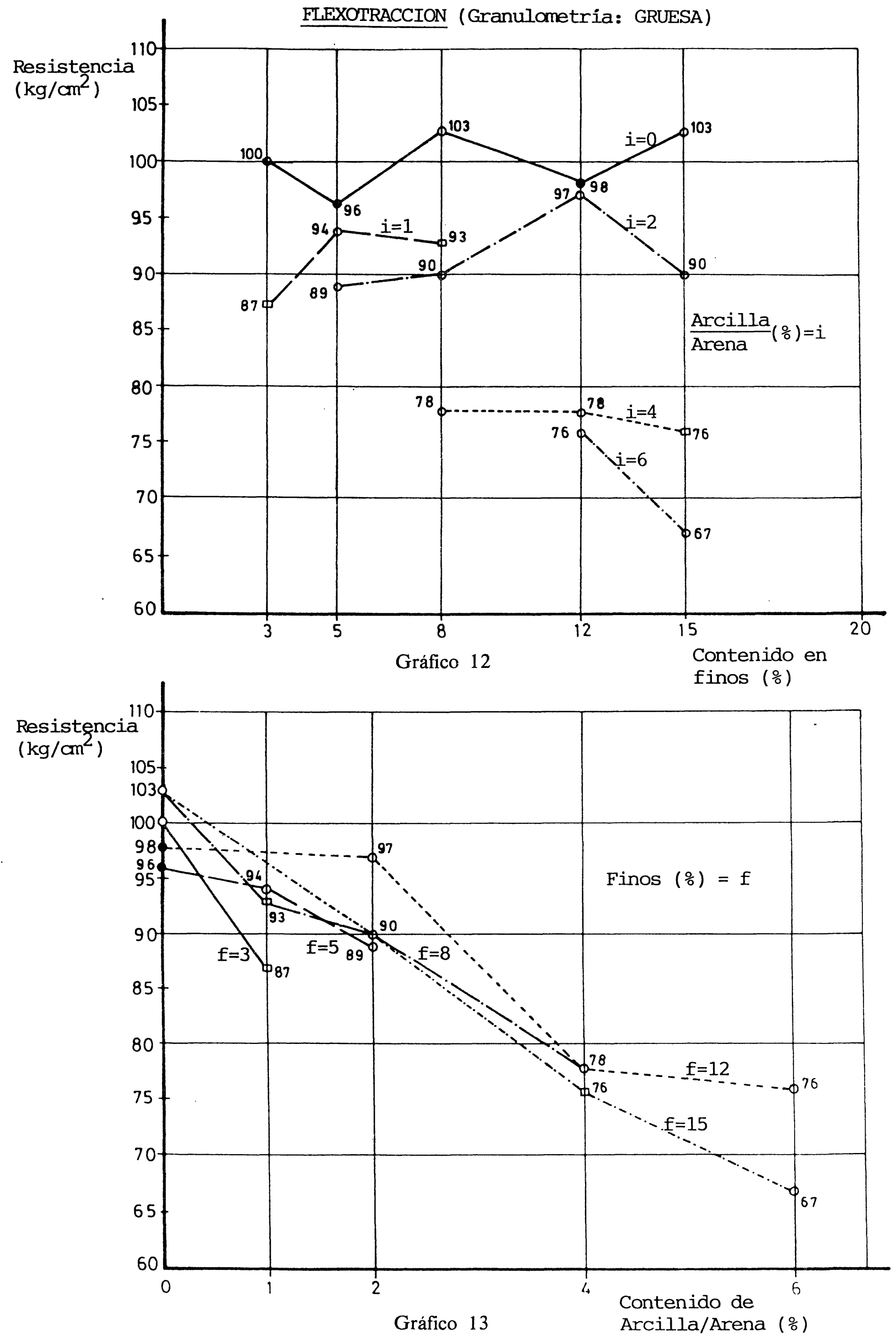

MATERIALES DE CONSTRUCCION. Vol. 35, n.॰200, octubre/noviembre/diciembre 1985 

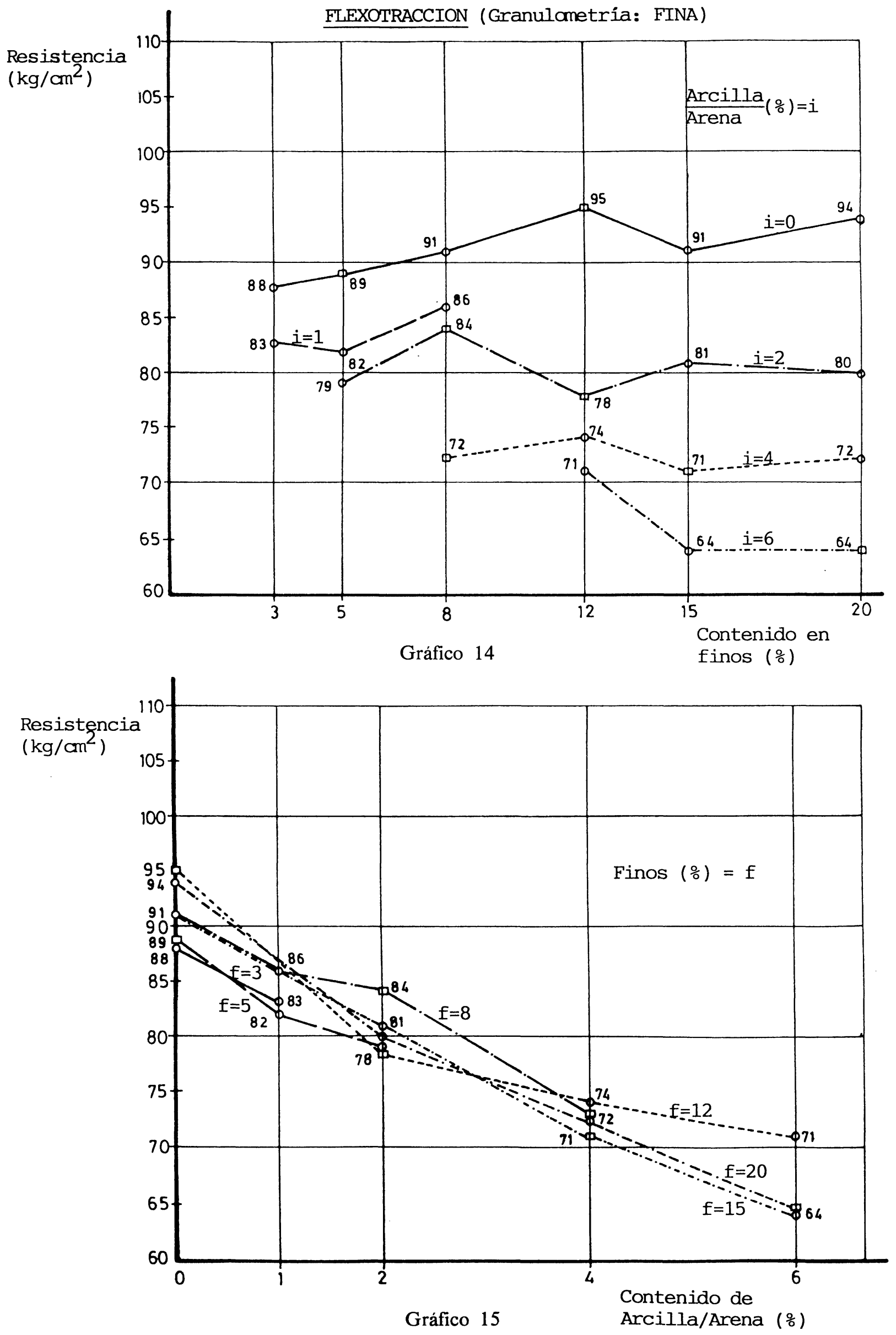


\section{CONCLUUSIONES}

En el presente trabajo se presenta una sola dosificación de cemento (cemento/arena $=1 / 4,2$ ). Se dispone también de resultados para dosificaciones $1 / 3$ y $1 / 6$ que sustancialmente conducen a análogas conclusiones.

Una constante en todo este trabajo ha sido el mantener la misma docilidad en todas las masas confeccionadas, como se ha indicado anteriormente.

Las conclusiones se pueden dividir en los siguientes apartados.

\subsection{Influencia de los finos calizos}

Cuando la proporción de arcilla en la arena es igual o menor al $2 \%$, se observa un incremento en las resistencias tanto de compresión como de flexotracción al aumentar el contenido de finos (gráficos $8,10,12$ y 14). Incluso para contenidos de $20 \%$ de finos se mantiene esta tendencia.

Para contenidos de arcilla mayores, las resistencias se estabilizan o disminuyen ligeramente. Estos efectos se corresponden en manera inversa en la disminución de la relación $\mathrm{A} / \mathrm{C}$.

La mejora de la plasticidad con el añadido de finos será la causa de este incremento de resistencia.

En los casos de ausencia de arcilla (relación arcilla/arena $=0$ ) se observa un incremento importante de las resistencias a compresión entre los valores 5 y $8 \%$ del contenido de finos, presentando a partir de este valor una tendencia ya sólo ligeramente ascendente. Puede pensarse pues que la cifra óptima estará por encima del $8 \%$. En la resistencia a flexotracción se observan más dispersiones y esta tendencia es menos acusada.

\subsection{Influencia del contaminante arcilloso}

La arcilla hace disminuir de forma importante la resistencia a compresión y flexotracción de los

CUADRO 3

Disminuciones medias de resistencia con la arcilla (\%)

\begin{tabular}{|c|c|c|c|c|}
\hline & \multicolumn{2}{|c|}{ Compresión } & \multicolumn{2}{|c|}{ Flexotracción } \\
\hline arcilla en & Granul. gruesa & Granul. fina & Granul. gruesa & Granul. fina \\
\hline 1 & 4 & 8 & 9 & 6 \\
\hline 2 & 11 & 19 & 9 & 13 \\
\hline 4 & 27 & 33 & 24 & 23 \\
\hline 6 & 38 & 41 & 29 & 29 \\
\hline
\end{tabular}


morteros ensayados (gráficos $9,11,13$ y 15).

Las disminuciones de resistencia medias, respecto a las obtenidas sin arcilla puede verse en el Cuadro 3.

La pérdida de resistencia con la arcilla es superior al incremento beneficioso del aumento de finos calizos. Asi un aumento del $2 \%$ en el contenido de arcilla de la arena influye sobre la variación de la relación $\mathrm{A} / \mathrm{C}$ tanto o más que un aumento del contenido en finos del $3 \%$ al $20 \%$ (gráficos 6 y 7). Se observa además que esta disminución de resistencia afecta más a la compresión que a la flexotracción.

\subsection{Influencia de la granulometría}

La granulometria fina como era de esperar ha producido descensos de resistencia tanto a compresión como a flexotracción.

Los descensos de resistencia medios entre iguales niveles de arcilla, ha sido del $13 \%$ para la resistencia en compresión y un $9 \%$ en flexotracción.

El emplear una granulometría gruesa en vez de fina produce una mejora en la resistencia para iguales condiciones de plasticidad. La importancia del efecto de la granulometria, en la resistencia, a veces despreciado, se traduce en la presente experimentación en ser equivalente a una variación del contenido de la arcilla en la arena del 1,5\%, valor ciertamente importante.

\subsection{Relaciones entre el equivalente de arena y las resistencias}

Ante la ausencia de normativa española referente al equivalente de arena, se ha tenido en cuenta la norma francesa NF P 18-301 que exige al equivalente de arena "a vista" en arenas provinientes de machaqueo un valor mayor de 65. En nuestro caso se ha utilizado el método "a pistón", siguiendo la normativa española actual. Suponemos que un valor de 60 sea equivalente al limite anterior.

Para cubrir esta exigencia, y de acuerdo con los resultados obtenidos, seria necesario que el contenido de finos no pasara del 13 ó $16 \%$ (gráficos 4 y 5), dependiendo de la granulometria (gruesa o fina), siempre que el contenido de arcilla fuera igual o inferior al $1 \%$.

Si se elevara esta última cifra al $2 \%$ los limites serian del 9 ó $14 \%$ de finos.

Según ésto, se deduce que la granulometría fina admite mayor contenido de finos que la gruesa, estando dentro de la exigencia de la norma francesa.

Si se considerara únicamente el valor del equivalente en arena (60) para discernir la aceptabilidad o rechazo de una muestra de arena, se admitirian implicitamente descensos de resistencia del orden de un $6 \%$ en la granulometria gruesa y de un $13 \%$ en la fina con respecto a los valores obtenidos con el $0 \%$ de arcilla y finos dentro de normas ( 3 y $5 \%$ ).

Además se rechazarian arenas que con un $2 \%$ de arcilla tienen contenidos altos de finos totales, cuando estas últimas muestras han dado lugar a mayores resistencias que las arenas con menor contenido en finos totales, que habrian sido aceptados.

Por otra parte, a igualdad de equivalente de arena los valores de resistencia son inferiores en aquellas muestras cuya disminución del citado valor, ha sido producido por la arcilla en vez de por los finos calizos. 
Igualmente el valor del equivalente en arena es muy sensible al contenido de arcilla. No parece que pueda caracterizarse con precisión la proporción de finos arcillosos admisible, acudiendo solamente a la determinación del equivalente en arena. En los gráficos 4 y 5 puede verse la importante variación del equivalente en función únicamente de la granulometría de la arena.

Por las razones expuestas no parece conveniente limitar la calificación de las arenas teniendo únicamente como dato el equivalente de arena, o el contenido de finos. Otros ensayos complementarios como el coeficiente de actividad o mejor el valor de azul de metileno, que se están estudiando, han de añadirse a los citados para evaluar la admisibilidad o rechazo de la arena.

\subsection{Conclusiones de esta fase en relación con una propuesta de modificación de normas sobre arenas}

Este estudio de morteros es a su vez preliminar a otro más amplio sobre hormigones, en ejecución. Sin embargo ya se pueden obtener algunos datos de interés en torno a los contenidos en finos, que pueden servir para una modificación de la norma E.H-82 actualmente en vigor.

La arcilla resulta muy perjudicial para la resistencia tanto a tracción como a compresión de los morteros de cementos. En cambio los finos calizos, hasta determinados valores pueden incluso ser hasta beneficiosos. De esto se deduce que es importante garantizar una limitación en el contenido de arcilla, vía equivalente de arena y azul de metileno tal como lo hace la norma francesa NF P 18-301 de Diciembre de 1983. De esta manera se podrán admitir porcentajes de finos calizos superiores a los de la actual norma española (5\%).

Los resultados obtenidos hasta el presente permiten intuir que a la terminación de las pruebas previstas para hormigones, se podrá situar el límite de finos calizos entre el 12 y el $15 \%$ siempre que se garantice unos contenidos bajos de arcilla.

De acuerdo con los gráficos $8,10,12$ y 14 los morteros confeccionados con contenidos de finos de ese orden, dan mejores resistencias en masas de análoga plasticidad, que los fabricados con arenas dentro de la norma actual (5\% de finos).

\section{REFERENCIAS}

(1) J. L. RAMiREZ, J. M. BARCENA, J. I. URRETA: "Panorama de las canteras de árido calizo en el Pais Vasco". Cemento y Hormigón. Noviembre 1984.

(2) J. L. RAMIREZ, J. M. BARCENA, J. I. URRETA: "Caracteristicas fisicas y quimicas de las arenas calizas de las canteras del Pais Vasco". Hormigón y Acero, n. ${ }^{\circ} 154$ - 1. ${ }^{\text {er }}$ Trimestre 1985.

(3) J. L. RAMIREZ, J. M. BARCENA, J. I. URRETA: "Influencia de los finos calizos y arcillosos en la resistencia de morteros de cemento". Monografia LABEIN - Octubre 1984.

(4) J. L. RAMIREZ, J. M. BARCENA, J. I. URRETA: "Estudio sobre la nocividad y corrección de los finos de las arenas calizas de machaqueo para hormigones en el Pais Vasco". Monografia LABEIN - Septiembre 1983. 